Review Article

\title{
Thioflavin-T: A Versatile Optical Probe for Chemo and Biosensing
}

\author{
AAFRIN M PETTIWALA ${ }^{1}$, NIYATI H MUDLIAR ${ }^{1}$ and PRABHAT K SINGH $^{1,2, *}$ \\ ${ }^{1}$ Radiation and Photochemistry Division, Bhabha Atomic Research Centre, Mumbai 400 085, India \\ ${ }^{2}$ Homi Bhabha National Institute, Anu Shaktinagar, Mumbai 400 094, India
}

(Received on 21 March 2018; Accepted on 15 September 2018)

\begin{abstract}
Sensing of chemical and biological molecules using extrinsic fluorescence probes has gained a lot of attention in contemporary research due to their high sensitivity and selectivity towards wide array of analytes. In this review article, we have focused on the photophysical properties of Thioflavin- $T$ and their subsequent modulations in presence of various different class of analytes which enable sensitive detection of different molecules. The versatility of Thioflavin-T, as fluorescent probe, is well exhibited by its ability to interact with various molecules which, in turn, act as direct or indirect platforms for detection of both biological and chemical molecules. Through, this short review, we aim to encourage other researchers to further explore the interesting photophysical properties of Thioflavin-T for sensing other clinically important biological and chemical analytes.
\end{abstract}

Keywords: Thioflavin-T; Fluorescence Sensor; Ultrafast Molecular Rotor; Bio-Sensor, Turn On/Turn Off Sensors; Thioflavin-T Aggregates; Thioflavin-T Photophysics

\section{Introduction}

Devising sensitive and selective fluorescence sensor platforms for precise detection of chemical and biological analytes holds great relevance in bioanalytical and biomedical research industries (Amdursky et al., 2012; Yao et al., 2014). A plethora of fluorophores have been employed for chemo and biosensing applications in recent times. Owing to the sensitive photophysical response of the fluorescent dyes towards their micro-environment, these photophysical parameters of the dye molecules can easily be tweaked to allow sensing of various analytes (Amdursky et al., 2012; Biju, 2014).

One such cationic dye which has been extensively used as fluorescent probe for sensing wide variety of analyte is Thioflavin-T. Thioflavin-T (ThT) belongs to the class of ultrafast molecular rotor dye, which has the inherent ability to undergo the nonradiative ultrafast bond twisting process around single $\mathrm{C}-\mathrm{C}$ bond (Scheme 1) that makes this molecule practically non-emissive in free state and in solvents of low viscosity (Haidekker et al., 2010; Singh et al., 2010a). However, in a highly viscous media or in a restricted micro-environment, the non-radiative ultrafast bond twisting process is suppressed, as a consequence of which, ThT exhibits fluorescence enhancement by several orders of magnitudes (Singh et al., 2010a; Singh et al., 2010b). This characteristic increase in fluorescence intensity of ThT forms the basis of its sensory mechanism for wide array of analytes like amyloid fibrils, DNA (Murudkar et al., 2012), heparin (Mudliar and Singh, 2016a), metal-ions (Ge et al., 2014; Wang et al., 2011) and so on.

In this short review article, we provide a detailed account of photophysical properties of ThT and its application as a fluorescent sensor. Apart from the routine usage of ThT as an amyloid sensor (Chu et al., 2007; Maezawa et al., 2008; Singh et al., 2015a), it also facilitates sensing of G-quadruplex DNA which are commonly implicated in important cellular functions (Faverie et al., 2014). The ThT-G-quadruplex scaffold acts as a flexible platform for detection of both chemical and biological moieties (Ge et al., 2014; Tong et al., 2013). Very recently, ThT has been also reported to sense an important bioanalyte, Heparin, which is the most widely used blood anticoagulant in clinical applications (Mudliar and Singh, 2016a). Further, we

*Authorfor Correspondence: E-mail: prabhatk@barc.gov.in; prabhatsingh988@gmail.com 
have also provided a brief account of the supramolecular interactions between ThT and macrocyclic host molecules which have been further used for efficient and selective sensing of proteins (Pettiwala and Singh, 2018a) and amino acids (Pettiwala and Singh, 2017). Thus, through this short account, we aim to provide the key sensory applications of Thioflavin-T in chemo and bio-sensing field.

\section{Photophysics of Thioflavin-T}

To understand the mechanism behind the distinctive fluorescence sensitivity of Thioflavin-T (ThT) towards its local microenvironment, the photophysical properties of ThT have been heavily investigated and debated in the literature. Various hypotheses have been put forward to explain the enormous increase in fluorescence intensity of ThT upon association with amyloid fibrils. One hypothesis which has been highly accepted and supported by both ab initio quantum chemical calculations and experimental evidences is that ThT belongs to the class of molecular rotor dyes. The organic dyes which have the ability to undergo an intramolecular twisting motion, in its excited state, are commonly described as molecular rotors (Amdursky et al., 2012; Haidekker et al., 2005; Haidekker et al., 2010; Rettig et al., 1994). By virtue of its molecular rotor property, the benzothiazole moiety of ThT undergoes a very fast rotation relative to dimethyl aminobenzene group in bulk water (Scheme 1). This ultrafast bond twisting process activates an efficient non-radiative decay channel for the molecule which results in a very low emission yield (Amdursky et al., 2012; Voropai et al., 2003). On the other hand, in highly viscous solutions, the ultrafast bond twisting process of ThT is significantly impeded which leads to a drastic enhancement in fluorescence intensity of the dye (Amdursky et al., 2012; Singh et al., 2010a).

During the past few years, a remarkable number of spectroscopic investigations, along with high level of quantum chemical calculations have provided proofs to understand the origin of fluorescence enhancement of ThT and its molecular rotor nature. The molecular rotor nature of ThT was first proposed by Voropai et al. which was then supported by quantum chemical calculations of ThT dye contributed by Stsiapura and others (Stsiapura et al., 2007;

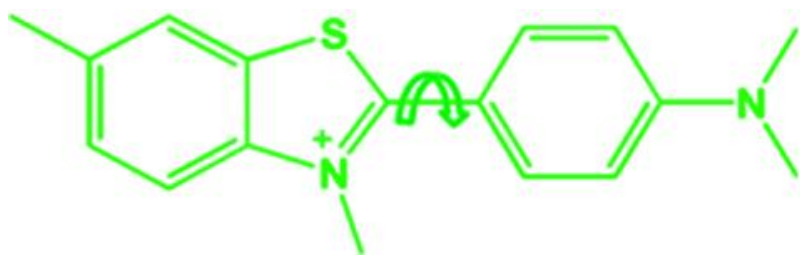

Scheme 1: Molecular structure of ThT

Stsiapura et al., 2008; Stsiapura et al., 2010; Voropai et al., 2003). These calculations led to the proposal that internal rotation around single $\mathrm{C}-\mathrm{C}$ bond between benzothiazole and dimethyl aminobenzene moiety (Scheme 1) is responsible for non-radiative decay of ThT. Moreover, the formation of charge transfer state, due to the internal rotation between the benzothiazole and dimethyl aminobenzene rings, is favourable in energy. However, the first experimental proof for its molecular rotor nature was provided by ultrafast fluorescence spectroscopic measurements which directly measured the rates for the bond twisting process in water and solvents of varying viscosity (Singh et al., 2010b; Singh et al., 2015a; Srivastava et al., 2010). To further understand the molecular structure and various conformers of ThT, quantumchemical calculations in ground and excited state of ThT were investigated as a function of dihedral angle of C-C bond (Fig. 1A) (Amdursky et al., 2012; Singh et al., 2010a; Singh et al., 2010b; Stsiapura et al., 2008). These calculations suggested that ThT in ground state $S_{0}$ has non-planar conformation due to presence of methyl group on nitrogen atom of benzothiazole ring which renders ThT molecule in strictly non-planar conformation. The torsional angle $\varphi$ between benzothiazole and aminobenzene rings was found to be $\sim 37^{\circ}$ at which potential energy minima was obtained. While, the minimum in potential energy, for ThT, in the excited singlet state $\left(\mathrm{S}_{1}\right)$, is obtained at $\varphi \sim 90^{\circ}$ and there is no energy barrier between two states $\varphi \sim 37^{\circ}$ and $\varphi \sim 90^{\circ}$. Thus, upon photo-excitation, ThT undergoes ultrafast bond twisting process to move from radiative locally excited (LE) state (quasi minimum state), at dihedral angle $\varphi \sim 37^{\circ}$, to nonradiative twisted internal charge transfer (TICT) state (global minimum state), at dihedral angle $\varphi \sim 90^{\circ}$ (Singh et al., 2010a). This transition from LE to TICT state is accompanied by a drop in oscillator strength from initial value of 1.1 at $\varphi \sim 37^{\circ}$ to 0.01 at $\varphi \sim 90^{\circ}$ (Fig. 1B) (Singh et al., 2010a). 

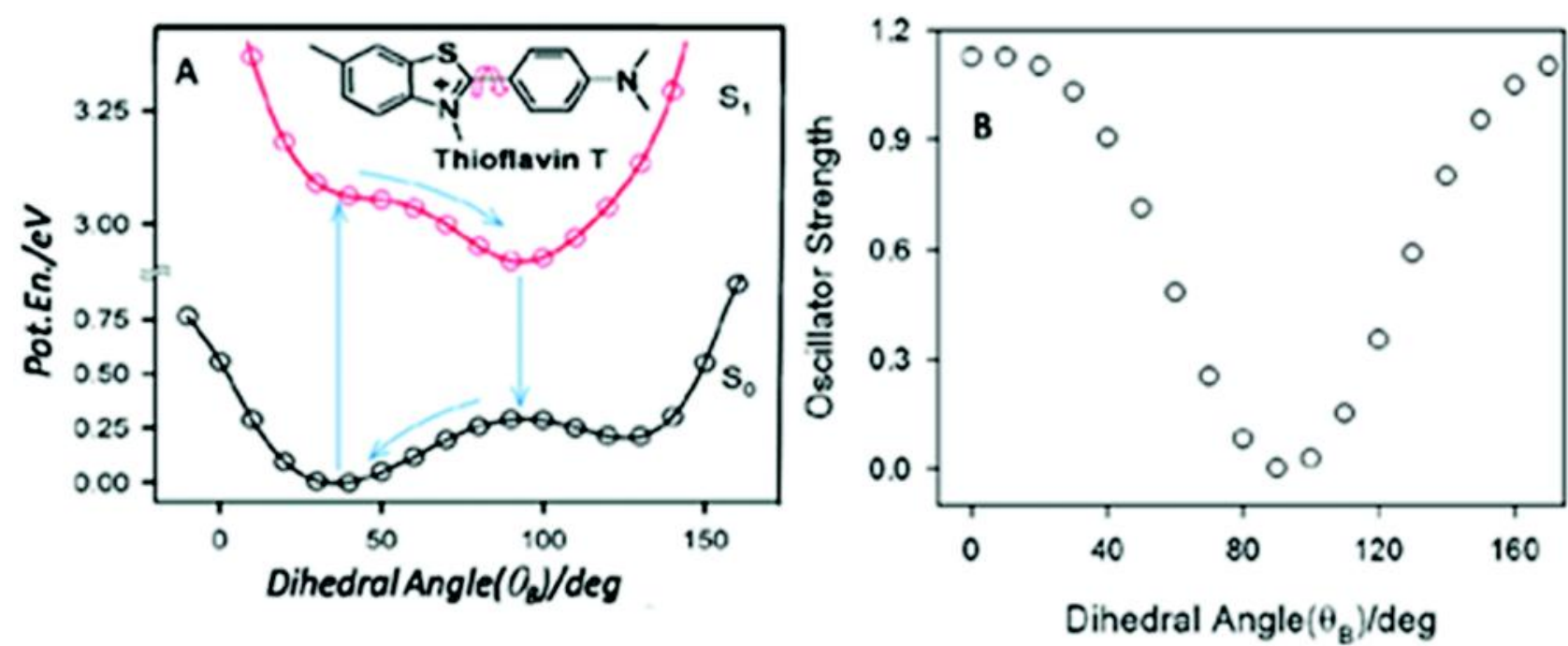

Fig. 1: A) Ground-state $\left(S_{0}\right)$ and excited-state $\left(S_{1}\right)$ potential energies of ThT as a function of dihedral angle $\theta_{B}$. (B) Variation in the oscillator strength with the dihedral angle $\theta_{B}$ for the $S_{0}-S_{1}$ transition of ThT in aqueous solution. (Adapted from Singh et al. 2010 with permission Copyright (2010) American Chemical Society)

Based on these calculations, a scheme for photophysical processes in the excited state of ThT was proposed. According to this scheme (Fig. 2A), a twisted intramolecular charge transfer state (TICT) is achieved by the ThT molecule, in its excited singlet state, which results in the change of dihedral angle around central single $\mathrm{C}-\mathrm{C}$ bond from $37^{\circ}$ to $90^{\circ}$ accompanied by transition from fluorescent LE state to non-fluorescent TICT state. This LE $\rightarrow$ TICT transition competes with radiative transition from $\mathrm{LE}$ state and accounts for significant quenching of ThT in aqueous solution. On the other hand, in highly viscous solvents, the internal rotation between aromatic rings of ThT is blocked and hence the transition from LE $\rightarrow$ TICT is suppressed, which subsequently leads to high fluorescence yield of ThT (Stsiapura et al., 2007). Further studies, using femtosecond transient-absorption technique, by Stsiapura et al. revealed a modified scheme wherein non-radiative deactivation process proceeds through a conical intersection between TICT $\left(\mathrm{S}_{1}\right)$ and $\mathrm{S}_{0}$ energy levels in concordance with earlier proposed scheme wherein ThT behaves as a molecular rotor (Stsiapura et al., 2010) (Fig. 2B).

In conjunction with quantum chemical calculations, extensive experimental studies were done to understand the effect of solvent viscosity,
(A)

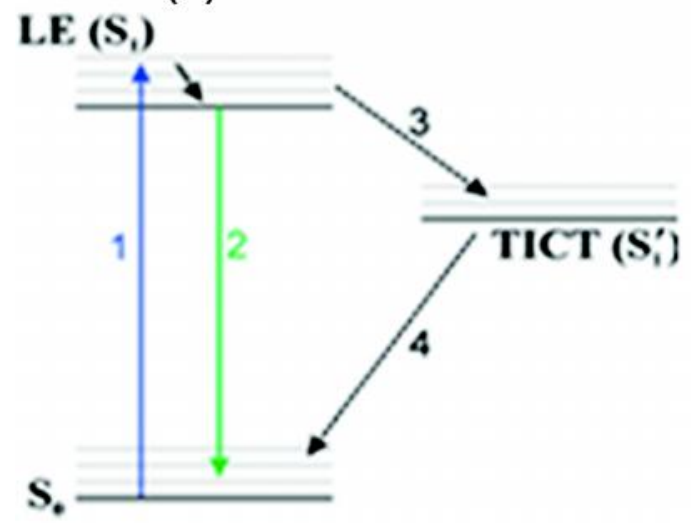

(B)

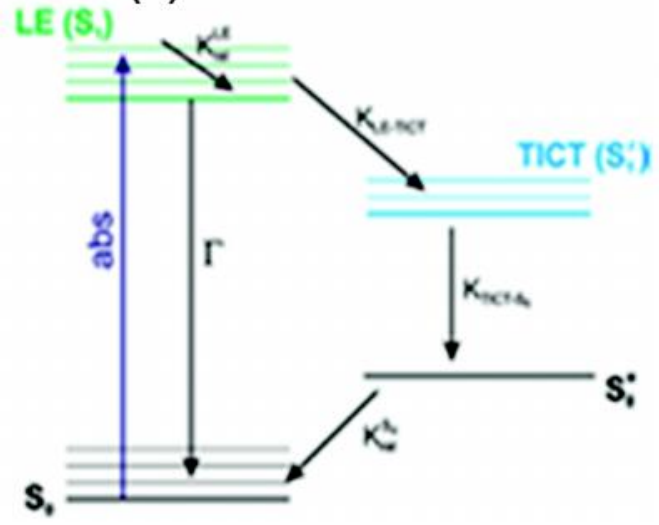

Fig. 2: (A) Scheme of excitation energy deactivation in ThT molecule (Adapted from Stsiapura et al., 2007 with permission Copyright (2007) American Chemical Society) and (B): Modified excitation energy deactivation scheme of ThT (Adapted from Stsiapura et al., 2010 with permission Copyright (2010) American Chemical Society) 
temperature, dielectric constant and pressure on photophysical properties of ThT. In 2003, it was first shown that solvent viscosity affects the spectral properties of ThT (Voropai et al., 2003), but only since 2007 a significant progress has been made in this domain. In subsequent studies by Maskevich et al., and Naik et al., it was established that ThT showed viscosity dependent emission with low degree of sensitivity towards solvent polarity (Maskevich et al., 2007; Naik et al., 2009). Moreover, it was shown that the photophysical response of ThT can be modulated by altering the viscosity of solution, such as with acetonitrile-ethylene glycol and glycerol-water solvent mixtures (Stsiapura et al., 2008; Sulatskaya et al., 2012; Sulatskaya et al., 2010). In 2008, Stsiapura et al. demonstrated that decrease in glycerol viscosity induced by heating or varying glycerol-water ratio led to a dramatic reduction in fluorescence yield and decrease of the average decay lifetime of ThT emission (Stsiapura et al., 2008). Huppert et al. studied the non-radiative process of ThT in 1-propanol as a function of temperature, wherein it was found that non-radiative decay rate decreased by three orders of magnitude when temperature was lowered to $88 \mathrm{~K}$ (Amdursky et al., 2011a). The reduction in nonradiative rate, upon decreasing temperature, was attributed to increase in viscosity by $\sim 2.5$ orders of magnitude (Amdursky et al., 2011a). These findings are in concordance with previous works, where it has been described that ThT, in highly viscous solution, exhibits high fluorescence intensity, and hence large reduction in the non-radiative rate processes (Singh et al., 2010b). Similarly, a change in hydrostatic pressure was shown to affect the viscosity and dielectric constant of solution, which in turn, modulates the photophysical properties of ThT (Amdursky et al., 2011b). Photophysical properties of ThT have also been investigated in various confined media like glass matrix (Schirra, 1985), polymer (Raj and Ramaraj, 2001), nano-confined water pool (Singh et al., 2009; Singh et al., 2011b; Singh and Nath, 2012), ionic liquids (Singh et al., 2015b; Singh et al., 2016), micelles (Kumar et al., 2008; Singh and Nath, 2013) and so on. In all these confined media, ThT fluorescence is highly dependent on local microviscosity. Thus, both experimental studies and quantum chemical calculations illustrate that ThT exhibits typical characteristic of molecular rotors described by ultrafast bond twisting process and its photophysics is highly sensitive to the local micro-environment.

\section{Thioflavin-T: Amyloid Fibril Sensor}

In 1959, ThT was first described as a potent amyloid fibril marker. Since then,ThT has emerged as a gold standard probe for detection of amyloid fibrils (Groenning et al., 2007b; LeVine, 1993; RodrýiguezRodrýiguez et al., 2009; Sabate and Saupe, 2007). The distinct increment in fluorescence intensity of ThT, on interaction with amyloid fibrils or amyloid like fibrils, makes it the most convenient diagnostic probe for detection of amyloid fibrils. Apart from acetylcholinesterase (Harel et al., 2008) and serum albumins (Sen et al., 2009), ThT does not associate with globular proteins in native state or amorphous aggregates of proteins, thus making it highly specific probe for amyloid fibrils (LeVine, 1993; Vetri et al., 2007). Amyloid fibrils are insoluble, disordered proteinaceous aggregates, rich in $\beta$-sheet structure, which are associated with various neurodegenerative disorders like Parkinson's disease, Alzheimer's disease and transmissible spongiform encephalopathies etc. (Biancalana and Koide, 2010; Chiti and Dobson, 2006; Gestwicki et al., 2004; Singh et al., 2015a). Thus, sensing of amyloid fibrils and tracing the fibrillation process becomes important from medical point of view. The applicability of ThT is not only limited to simple sensing of amyloid fibrils, but has also been extended to elucidate the mechanism of fibril formation and to study the structure of fibrils.

Although it is well established fact that ThT is an excellent tool for sensing amyloid fibrils, the mechanism of its binding to amyloid fibrils, and the reason behind observed changes in its photophysical properties are still ambiguous. The observed fluorescence enhancement of ThT on interaction with amyloid fibrils has been previously attributed to formation of excimers (Groenning et al., 2007a), micelles (Khurana et al., 2005), dimers or aggregates (Groenning et al., 2007b) of ThT in the fibrillar medium. However, these propositions stand weak as the optical microscopy results have demonstrated that ThT interacts with amyloid fibrils in the monomeric form (Kitts and Bout, 2009). More importantly, the spectral properties ofThT aggregation, reported in a number of recent works, has clearly rejected the theory of ThT dimerization or aggregation in the fibrillar medium (Mudliar and Singh, 2016b; Singh et al., 


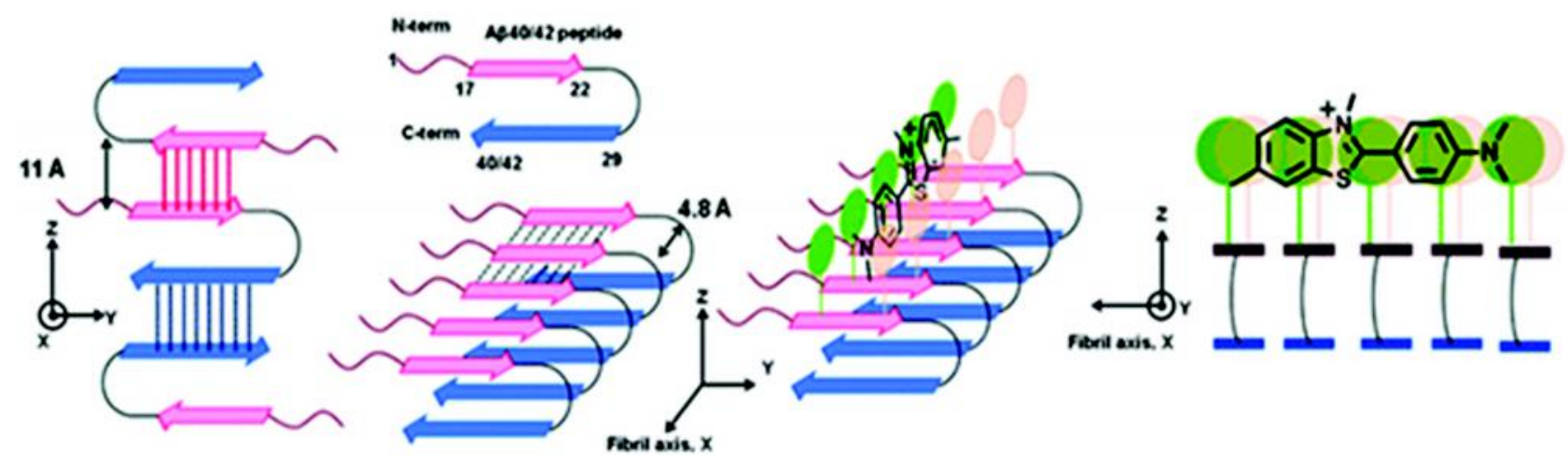

Fig. 3: Schematic representation of "Channel" binding model of ThT binding to fibrils (Adapted from Noel et al., 2013 with permission from Royal Society of Chemistry)

2015a). Thus, the mechanism behind fluorescence enhancement of ThT, upon incorporation into amyloid fibrils, is the restriction of ultrafast bond twisting process around single $\mathrm{C}-\mathrm{C}$ bond in the excited state of ThT, which is strongly supported by experimental evidences as described earlier in the article.

Another aspect of ThT-amyloid fibril interaction, which is highly investigated, is the binding mode of ThT to amyloid fibrils. ThT specifically recognizes cross- $\beta$ structures in amyloid fibrils, which form specific arrangement of side chains referred to as cross-strand ladders (Biancalana and Koide, 2010; Groenning et al., 2007a; Noel et al., 2013). These cross-strand ladders contain repetitive side-chain interactions running across $\beta$-strands in $\beta$-sheets which are parallel to the long axis of the fibril (Biancalana and Koide, 2010; Ivanova et al., 2009; Nelson and Eisenberg, 2006). Krebs et al., using polarized fluorescent microscopy, proposed that ThT binds along surface side-chain grooves which run parallel to long-axis of $\beta$-sheet that also consist of cross-strand ladders (Fig. 3). This binding model of ThT to amyloid fibrils is referred to as Channel binding model (Biancalana and Koide, 2010; Krebs et al., 2005). Thus, ThT interacts with a common structural feature of amyloid fibrils irrespective of protein type, be it Alzheimer's related fibrils (Maezawa et al., 2008) or prion associated amyloid fibrils (Sabate et al., 2008) or fibrils of generic protein like BSA (Mudliar et al., 2016). Importantly, ThT has been routinely employed in in vitro assays and kinetic studies of amyloid fibril formation, which not only gives insights into the molecular mechanism behind amyloid fibrillation process, but also aids in identifying potential inhibitors of protein fibrillation process. Interestingly, very recently, using ultrafast fluorescence spectroscopy, it has been shown that apart from the channel binding mode, a large fraction of fibril bound ThT, also occupies surface binding sites on the fibril (Singh et al., 2015a). Interestingly, these surface bound ThT molecules do not contribute to the characteristic emission enhancement observed for ThT in the fibrillar medium (Singh et al., 2015a).

Although ThT displays diverse applicability in association of amyloid fibrils, but it cannot be employed for in vivo imaging due to its positive charge which makes it hydrophilic and prevents it from entering blood brain barrier (Klunk et al., 2001). Additionally, the unaltered spectral position of ThT in association with amyloid fibrils further limits its applications in imaging studies (Maskevich et al., 2007; Mora et al., 2016; Singh et al., 2010a). Thus, recently researchers have focused on developing neutral derivatives of ThT which displays prominent spectral changes on association with amyloid fibrils for the efficient detection of amyloid fibrils. One such work has been demonstrated, wherein a neutral derivative of ThT was synthesized 2-[2'-Me,4'-(dimethylamino)phenyl] benzothiazole (2Me-DABT) and its interaction with insulin fibrils was studied using time-resolved and steady-state fluorescence techniques (Mora et al., 2016). Upon addition of insulin fibrils to aqueous solution of $2 \mathrm{Me}-\mathrm{DABT}$, a significant increase in emission intensity was observed accompanied by a distinct hypsochromic shift in emission spectra. Such a large shift in emission spectra of 2Me-DABT, upon interaction with insulin fibrils, suggests its potential use as ratiometric sensor for amyloid fibrils, and it 
also provides a distinctive advantage in imaging applications due to relatively low background signal (Mora et al., 2016). Thus, ThT continues to be the most extensively utilized probe molecule for sensing amyloid fibrils with minor alterations, in order to find better and efficient methods to detect amyloid fibrils.

\section{Thioflavin-T: Nucleic Acid Sensor}

Nucleic acids (DNA/RNA) are one of the most crucial biomolecules present in living system, which monitor essential cellular process. The inevitable importance of nucleic acids and their non-canonical structures in functioning of living system has led to the development of various strategies to detect them (Cosnier and Mailley, 2008; Wu and Guo, 2014). Considering the significance of mutated DNA in fatal diseases like cancer, sensing of both natural DNA and disease related DNA has been targeted by researchers (Cosnier and Mailley, 2008). Some of the sensitive and efficient platforms developed for detection of nucleic acids are Polymerase Chain Reaction (PCR) (Schaerli et al., 2009; Tassone et al., 2008), DNA microarrays (G. Ventimiglia and S. Petralia, 2013; Sassolas et al., 2008), rolling circle amplification (RCA) (Ji et al., 2012), blotting methods (Glenn and Andreou, 2013; Huang et al., 2010), hybridization chain reaction (HCR) (Guo et al., 2016) and so on. Although these conventional techniques are commonly used, but they involve complex procedures, thus, limiting their utility in wider scenarios. Apart from these conventional techniques, extrinsic fluorescent probes have been highly investigated for their interaction with nucleic acids. ThT turns out to be a very efficient and facile probe molecule, among others, for detection of nucleic acid, owing to its unique fluorescence light up attribute on interacting with nucleic acids.

ThT has been identified as an amyloid fibril sensor for last 50 years, but now, it has recently been in focus for its interaction with nucleic acids/DNA (Ilanchelian and Ramaraj, 2004; Liu et al., 2013). ThT, upon interacting with DNA, displays a dramatic fluorescence enhancement, compared to other DNA binding dyes like ethidium bromide and 4'6diaminodino-2 phenylindole (Murudkar et al., 2012). The increase in fluorescence intensity of ThT, on association with DNA, is attributed to restriction of its intramolecular torsional relaxation. On binding to DNA, the ultrafast bond twisting process around central C-C bond in ThT, is suppressed and as a result the non-radiative deactivation channel is set off and fluorescence is switched on (Ilanchelian and Ramaraj, 2004; Murudkar et al., 2012). This remarkable increase in fluorescence intensity of ThT on binding to DNA led to tremendous research activity in constructing sensor platforms based on ThT for detection of different types of DNA.

Few studies, involving ThT and DNA, concentrate on the binding mechanism of ThT to DNA. One such study to probe the interaction between ThT and DNA was reported using steadystate and femtosecond time-resolved transient emission techniques (Murudkar et al., 2014). The alteration in spectral properties of ThT, on interaction with DNA, were found to be in concordance with earlier studies (Liu et al., 2013). However, the binding mode of ThT to DNA was found to be electrostatic interaction and intercalation, as indicated by salt, viscosity and resonance energy transfer studies (Murudkar et al., 2014). In another study, a combination of absorption and emission titrations under different conditions (salt content, temperature), fluorescence quenching, T-jump relaxation methods and viscosity experiments were employed to understand the mechanistic aspects of ThT-DNA interaction (Biancardi et al., 2014). The results pointed out that ThT in its monomeric form, undergoes intercalates between DNA base pairs. In addition to intercalation and electrostatic interaction as binding modes of ThT to DNA, it was observed that under ThT excess condition, ThT dimer bind to the DNA grooves as shown by QM/MM MD simulation (Biancardi et al., 2014). In 2017, the same group further explored the binding interaction between ThT and DNA using molecular dynamics and quantum chemical modelling (Biancardi et al., 2017).

ThT-DNA interaction has been exploited in various formats to construct sensors for secondary structural change in DNA or DNA structures implicated in disease conditions. One such study is employing ThT as sensor for studying premelting structural changes in DNA, wherein ThT was found to be sensitive to structural changes induced by temperature, in pre-melting regime, which remains undetected by other spectroscopic techniques like Circular Dichroism spectroscopy (Murudkar et al., 2012). Another interesting work, wherein ThT acts 
as a fluorescent probe to understand the interaction between imidazolium based ionic liquids and calf thymus DNA was reported in 2012 (Singh et al., 2012). The addition of ionic liquid to DNA bound ThT leads to a decrease in fluorescence intensity, indicating a displacement of ThT from DNA surface, owing to strong interaction between DNA and ionic liquids. This proposition was further supported by femtosecond fluorescence up conversion studies (Singh et al., 2012). Furthermore, ThT was employed to probe the interaction between DNA and ionic liquids of varying hydrophobicity which revealed that hydrophobicity of ionic liquids, in addition to electrostatic interaction, plays an important role in ionic liquid-DNA interaction (Singh et al., 2012).

In 2013 Liu et al. demonstrated that ThT was able to selectively recognize ds DNA containing cavity structures such as gap site, mismatch site or abasic site (Liu et al., 2013). These are commonly implicated in mutagenic DNA and carcinogenic lesions. Selective recognition of these cavity structures by ThT was illustrated by increase in fluorescence enhancement as compared to full matched DNA which showed comparatively negligible fluorescence (Fig. 4). This increase in fluorescence of ThT on associating with ds DNA cavity structures originated from restriction of its non-radiative torsional relaxation of single C-C bond (Liu et al., 2013). This ability of ThT to selectively recognize DNA cavity structures presents it as a potential platform for development of efficient and practical DNA sensors.

Natural self-assembled DNA/RNA structures such as G-quadruplexes and i-motif are commonly observed in the promoter regions of various human genes including proto-oncogene (RET) (Guo et al., 2007) and retinoblastoma ( $\mathrm{Rb})$ gene (Xu and
Sugiyama, 2006; Zhou et al., 2010). Rapid and convenient sensors for detection of these natural selfassembled DNA/RNA is highly important from the perspective of medical diagnostics. In 2015, ThT was employed as fluorescence sensor for detection of imotif in retinoblastoma $(\mathrm{Rb})$ and $\mathrm{RET}$ proto-oncogene using CD spectroscopy and fluorescence studies at different $\mathrm{pH}$ values. It was found that ThT acted as an efficient sensor to probe the conformational changes in i-motifs by the virtue of its high degree of sensitivity towards changes in its local microenvironment (Lee et al., 2015).

Another important self-assembled noncanonical structure formed by DNA/RNA is G-quadruplex. Gquadruplexes have gained widespread attention due to their potential applications in both in vitro and in vivo studies. These structures are often identified by the presence of signature G-tetrads formed by hydrogen bonding between four guanine residues which are further stabilized by Hoogsteen hydrogen bonding (Gellert et al., 1962; Phan et al., 2007). Gquadruplexes are involved in various biological functions like chromosome stability by blocking cellular polymerases and helicases, gene regulation, serving intermediates in recombination and telomerase activity. Sequences with potential to form G-quadruplex (G4) in human genome account for about $40 \%$ of all genes of which human telomeric regions contribute majorly. Apart from human telomeric regions, these sequences are encountered in the promoter regions of oncogenes like C-KIT, H-RAS, K-RAS, C-MYC, and also in untranslated regions of mRNAs, indicating their inevitable significance in proper functioning of biological systems and hence their precise detection is crucial (Faverie et al., 2014). There are various techniques which detect G4 structures like nuclear

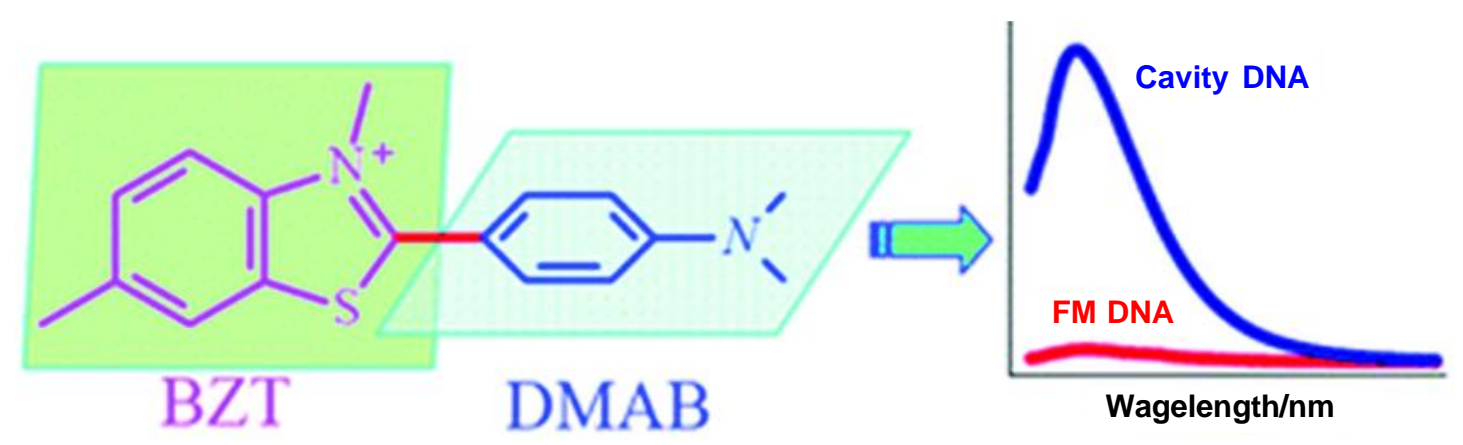

Fig. 4: Selective recognition of ds DNA cavities by ThT (Adapted from Liu et al., 2013 with permission from The Royal Society of Chemistry) 

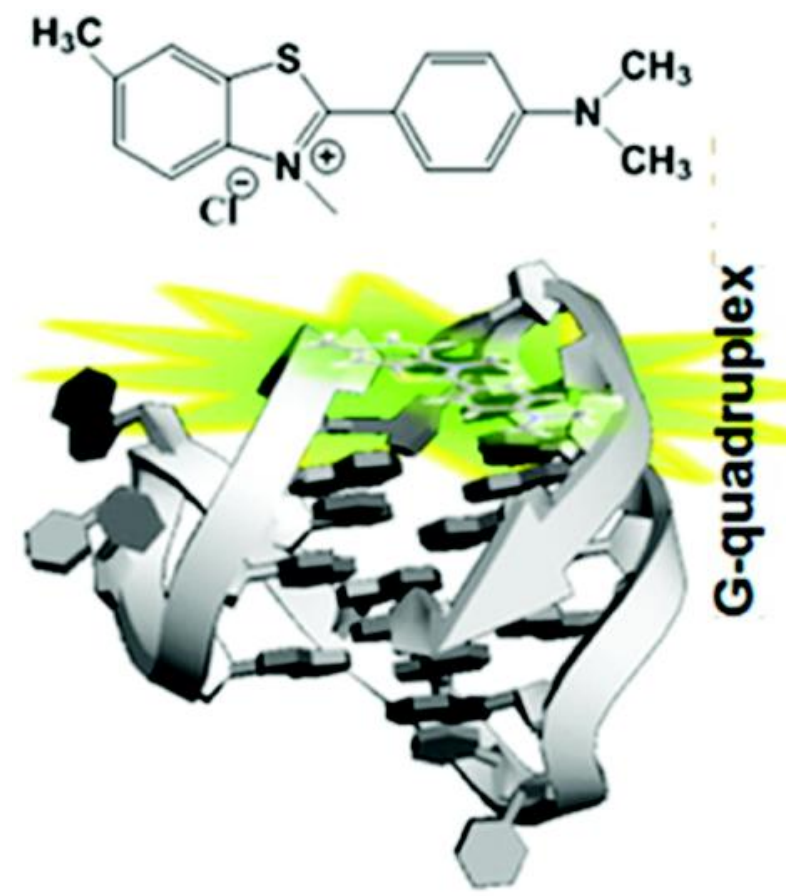

Fig. 5: Schematic representation of fluorescence turn-on of ThT in G-quadruplex (Adapted from Gabelica et al. 2013 with permission Copyright (2013) American Chemical Society

magnetic resonance (NMR), melting temperature determination (Mergny and Lacroix, 2009; Mergny et al., 2005), isothermal difference spectra and circular dichroism (CD). However, these techniques are time-consuming and require special expertise. Thus, there is a need for rapid and facile screening method for detection of G4 structure. It has been observed that, ThT becomes fluorescent in presence of G4 structures and hence acts as a sensitive label free probe for detecting G4 structures (Faverie et al., 2014; Gabelica et al., 2013) (Fig. 5).

ThT has been found to selectively induce and stabilize G4 structures (Mohanty et al., 2013). In 2013, the dual role of ThT was reported in inducing G4 structures in 22AG human telomeric DNA, and sensing the same through its fluorescence switch on mechanism attributed to the restriction of its ultrafast molecular rotor property (Mohanty et al., 2013). More importantly, it was noted that ThT, on interaction with other DNA forms (ss or duplex DNA and calf thymus DNA), resulted in relatively low emission enhancement, thus indicating the high specificity of ThT towards quadruplexes compared to other DNA forms. Additionally, a comparative study with thiazole orange (TO) interaction with G4, and other DNA forms, was performed wherein it was observed that TO showed similar fluorescence enhancement for all forms of DNA, indicating the lack of specificity towards G4 structures. Thus, ThT acts as a highly specific fluorescence sensor for G4 structures (Mohanty et al., 2013).

The increase in fluorescence of ThT, on associating with $\mathrm{G} 4$, is attributed to intercalation, groove binding and end stacking of ThT molecules which restricts the non-radiative ultrafast bond twisting process (Faverie et al., 2014; Mohanty et al., 2013). To further generalize and extend the application of ThT as fluorescent probe for detection of G4, a detailed screening of G4 forming sequence was carried out. It was distinctly noted that ThT displayed a drastic increase in emission intensity on association with G4 forming sequences, while in the case of control duplexes and single strands, relatively very less enhancement in emission intensity was observed for ThT (Fig. 6). Using a plate reader format hundreds of oligonucleotides with potential to form G4 structure were analysed (Faverie et al., 2014).

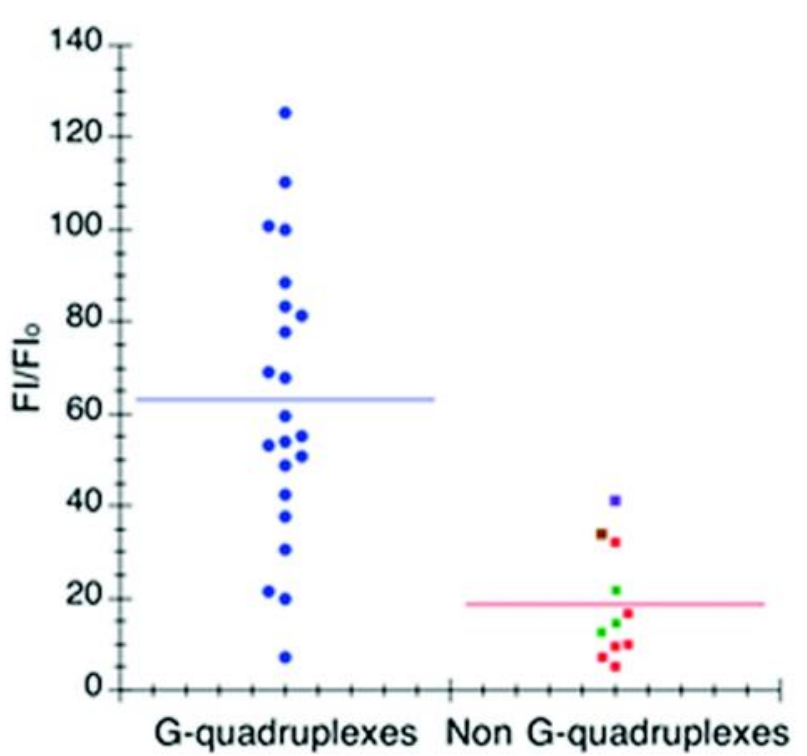

Fig. 6: Each point corresponds to fluorescence enhancement in the presence of a different oligonucleotide. The change in fluorescence emission is plotted for DNA and RNA quadruplexes on the left (in blue) and nonG-quadruplex structures on the right. Green dots correspond totrinucleotides, purple dot to parallelduplex, brown dot to the triplex and red to other nonquadruplex-forming sequences (Adapted from Faverie et al., 2014 with permission from Oxford University Press) 
Very recently, ThT has been demonstrated as an efficient fluorescent sensor for detection of RNA G-quadruplexes using fluorescence, absorption and emission lifetime studies (Fig. 7). ThT showcased a drastic fluorescence enhancement on interacting with RNA G-quadruplexes compared with other RNA forms (Xu et al., 2018b). This differential fluorescence response of ThT generated for G4 and non-G4 RNA structures renders ThT a simple and efficient sensor for RNA G-quadruplexes, in addition to DNA Gquadruplexes. The interesting and selective fluorescence properties showcased by ThT on associating with DNA G4 structures has allowed to devise facile and label-free fluorescence sensor platforms for detection for metal ions and biomolecules, which will be discussed in detail in the next section.

In 2014, Ge et al. reported a $\mathrm{Hg}^{2+}$ ions sensor platform based on ThT-DNA G-quadruplex framework. In this work, the interaction between ThT, $\mathrm{Hg}^{2+}$ ions and oligonucleotide sequences rich in guanosine and thymine loops was studied. $\mathrm{Hg}^{2+}$ ions are known to specifically interact with Thymine (T) rich oligonucleotides, thus, as speculated, $\mathrm{Hg}^{2+}$ ions interacted with the oligonucleotide, and blocked the process of G-quadruplex formation, which is observed in absence of $\mathrm{Hg}^{2+}$ ions (Ge et al., 2014). This blocking of G-quadruplex formation was reflected by decrease in fluorescence intensity of ThT which is normally high in the absence of $\mathrm{Hg}^{2+}$ ions due to formation of G4 structures. This sensor platform enabled sensitive and selective detection of $\mathrm{Hg}^{2+}$ ions with limit of detection (LOD) of $5 \mathrm{nM}$ (Ge et al., 2014).

Based on the similar principle of $\mathrm{Hg}^{2+}$ ions specific interaction with T-rich oligonucleotide sequences to form thymine-thymine mismatched base pair with $\mathrm{Hg}^{2+}$ ions ( $\left.\mathrm{T}-\mathrm{Hg}^{2+}-\mathrm{T}\right)$, a turn on fluorescent sensor for detection of $\mathrm{Hg}^{2+}$ ions was designed (Ono et al., 2011; Xu et al., 2018a). The sensors based on ThT-G4, reviewed so far, involve fluorescence quenching as signal output, which, in general, suffers from low sensitivity and is not a desirable feature. In this particular work, a turn on fluorescent sensor based on the strategy of proximity-dependent Gquadruplexes was devised, wherein in the absence of $\mathrm{Hg}^{2+}$ ions the 2 strands of G-rich DNA failed to form G4 structure resulting in negligible fluorescence intensity of ThT (Xu et al., 2018a). On addition of $\mathrm{Hg}^{2+}$ ions, a remarkable increase in ThT fluorescence was observed. This increase in fluorescence was attributed to formation of $\mathrm{G} 4$ assisted by $\mathrm{Hg}^{2+}$ ions which bridged the 2 strands of DNA by forming T$\mathrm{Hg}^{2+}-\mathrm{T}$ complexes. The LOD was found to be 10 $\mathrm{nM}$, and importantly, this sensor also enabled detection of $\mathrm{Hg}^{2+}$ ions in fetal calf serum (Xu et al., 2018a). Also, the ThT-G4 format has been utilised for selective recognition of $\mathrm{K}^{+}$ions with LOD of $1 \mathrm{mM}$. The sensor showed minimum specificity towards other tested metal ions (Liu et al., 2014).

In addition to metal ion sensing, ThT-G4 platform has also been explored for detection of various biomolecules. In one such work, a label-free turn on fluorescence sensor for detection of biothiols was constructed on ThT-G4 framework (Tong et al., 2013). The sensing approach is as follows: In the absence of oligonucleotide sequence ARGO100, ThT displays a weak fluorescence, however, upon addition of ARGO100, a remarkable increase in emission intensity is observed due to formation of G4 structure. Upon addition of $\mathrm{Hg}^{2+}$ ions, ThT-G4 complex is disrupted due to specific interaction between $\mathrm{Hg}^{2+}$ ions and thymine residues present in oligonucleotide. On

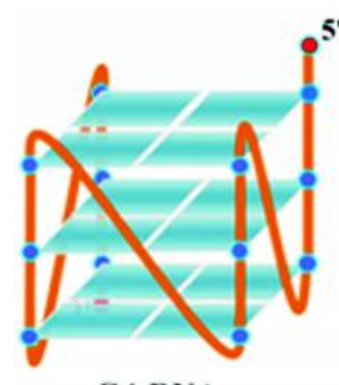

G4-RNA

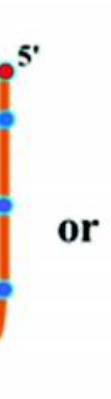

ss-RNA
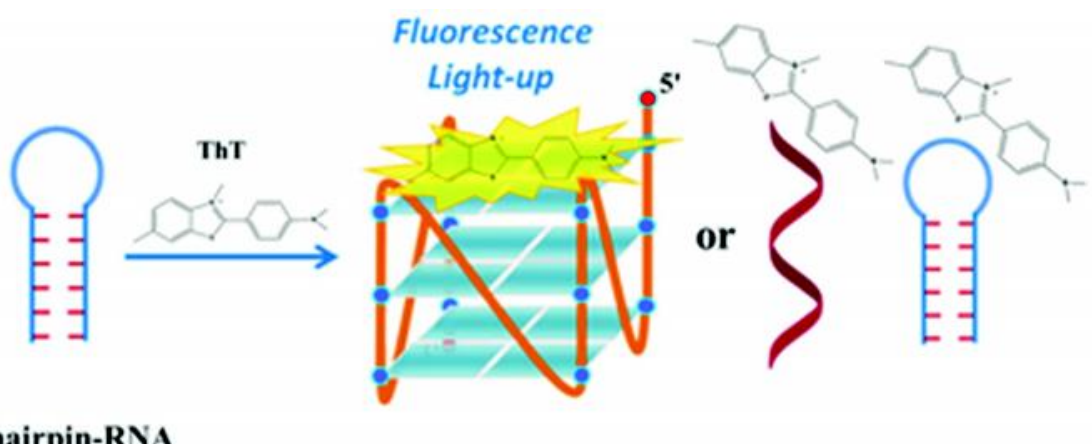

Fig. 7: Schematic representations of ThT binding with various RNA forms for G-quadruplex structure Recognition (Adapted from Xu et al., 2018b with permission from Nature Publishing Group) 
introducing biothiols (cysteine and glutathione), $\mathrm{Hg}^{2+}$ ions form stronger interaction with biothiols $(\mathrm{Hg}-\mathrm{S})$ as a result of which the G-quadruplex structure is restored and fluorescence enhancement is obtained. This sensor platform provides a sensitive mode for detection of cysteine and glutathione in the range of $2 \times 10^{-8}-2.5 \times 10^{-6} \mathrm{M}$ and $3 \times 10^{-8}-2 \times 10^{-6} \mathrm{M}$ with a LOD values $8.4 \mathrm{nM}$ and $13.9 \mathrm{nM}$ respectively (Tong et al., 2013). Based on a similar concept, detection method for $\mathrm{Ag}^{+}$ions and biothiols was developed using Gquadruplexes as a label free platform (Yang et al., 2016). This approach exploits the coordination of $\mathrm{Ag}^{+}$ ions with guanine, which does not allow the Gquadruplexes to form and leads quenched emission from ThT. However, when biothiols are added to this solution, $\mathrm{Ag}^{+}$ions are released owing to stronger coordination of $\mathrm{Ag}^{+}$ions and GSH leading to enhanced emission from ThT (Fig. 8). This method shows a good linear response for GSH in the range of 50$3600 \mathrm{nM}$ with a LOD value of $16 \mathrm{nM}$.

Liu et al. developed a Forster resonance energy transfer (FRET) based label free fluorescent sensor for detection of thrombin (Liu et al., 2015). The sensor system comprised of a conjugated polymer (CP) as energy donor while ThT as energy acceptor. In the absence of thrombin, ThT induced the transition from

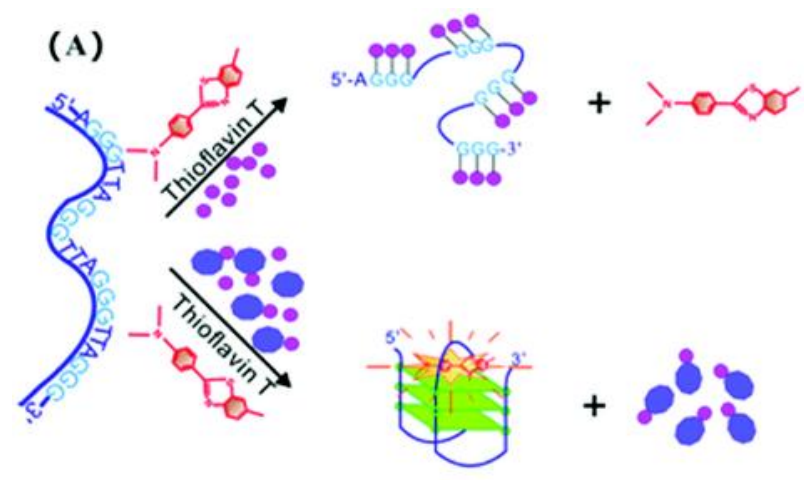

(B)

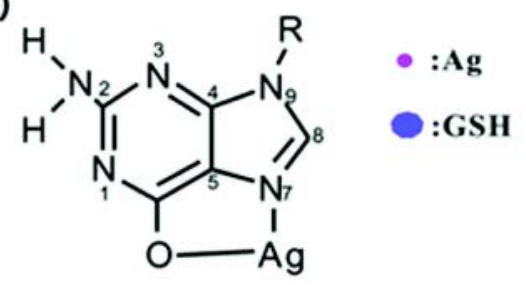

Fig. 8: Schematic illustration of the fluorescence changes of the ThT on interacting with Ag+ ions and biothiols (GSH) (Adapted from Yang et al., 2013 with permission from Royal Society of Chemistry) aptamer to G4 structure and resulted in fluorescence turn on signal. Due to electrostatic interactions between anionic $\mathrm{G} 4$ structure and cationic $\mathrm{CP}$, distance between the donor and the acceptor shortened and hence a high FRET signal was achieved. While in the presence of thrombin, aptamer forms a G4/thrombin complex first, followed by binding of ThT. Due to steric hindrance of thrombin, a long distance between the donor and acceptor exists, and hence a weak FRET signal is observed. Importantly, this sensor system allows rapid and sensitive detection of thrombin not only in aqueous solution but also in serum samples and hence presenting its potential utility in practical applications (Liu et al., 2015).

A label free and enzyme free biosensor for detection of liver cancer related short gene (MXR7) was devised based on target recycling, and ThT induced G4 formation in human serum(Li et al., 2015). The detection system consists of ThT and two hairpin DNA which contain domains showing affinity towards MXR7 and G-rich regions. The interaction between these domains, G-rich sequences and MXR7 results in formation of G-quadruplex which ultimately binds to ThT resulting in fluorescence enhancement. This sensor system facilitates sensitive detection of MXR7 in the range of $0 \mathrm{fM}$ to $350 \mathrm{fM}$ with LOD value of 10fM (Li et al., 2015).

Another sensor system based on ThT-G4 complexation was devised for detection of important biomolecule streptavidin. The sensor system consisted of target molecule streptavidin, ThT and G-rich biotinylated ssDNA (Bai et al., 2017). It was noted that in the presence of ThT, G-rich biotinylated ssDNA was transformed to form G-quadruplex DNA structure, which on association with ThT, resulted in high emission yield. In the absence of streptavidin, ThT-G-quadruplex DNA complex was digested by exonucleases resulting in release of ThT which exhibits weak fluorescence intensity. However, on addition of streptavidin the digestion of ThT-Gquadruplex DNA by exonuclease was prevented and thus the high emission yield was attained. The range of detection for streptavidin was found to be 0.05 $2500 \mathrm{ng} / \mathrm{ml}$ with low LOD value of $0.02 \mathrm{ng} / \mathrm{ml}$. Additionally the sensor also gave satisfactory results in 5\% human serum (Bai et al., 2017).

Thioflavin-T Interaction with Supramolecular Host: Sensor for Various Chemical and Biomolecules 
The modulation in photophysical properties of ThT is observed when placed in confined spaces like amyloid fibrils, water nanopools of reverse micelle, micelles, DNA, etc. One such confined space which have been highly studied for their interaction with ThT, and has been further propagated as a sensory platform for various biomolecules, are supramolecular host. One such class of supramolecular host is cyclodextrin. The interaction of ThT with cyclodextrins has been studied in detail using steady-state and femtosecond resolved emission measurements (Singh et al., 2011a). It has been shown that the inclusion of ThT by bcyclodextrin cavity leads to a sizeable increase in emission intensity consistent with the molecular rotor picture of ThT (Singh et al., 2011a). While $\beta$-CD leads to a sizeable complexation with ThT, $\alpha-\mathrm{CD}$, owing to its smaller cavity size, fails to form any complex with ThT, (Singh et al., 2011a) and contrastingly, $\gamma-\mathrm{CD}$ is able to form both 1:1 complex, (Murudkar et al., 2015) and 1:2 complex, (Singh et al., 2015a) where two ThT molecules are included, displaying an excimer like emission for ThT. Interestingly, sulfobutyl ether derivative of $\beta-\mathrm{CD}\left(\mathrm{SBE}_{7}-\beta-\mathrm{CD}\right)$ significantly improves the complexation of ThT, suggesting crucial role of extended hydrophobic cavities in determining the binding strength of ThT with this class of host molecules (Singh et al., 2015c). The 1:1 complex of $\gamma$-CD with ThT has been projected as a sensor for hydrocarbon chains, where significant emission enhancement is observed for ThT- $\gamma-\mathrm{CD}$ complex in the presence of hydrocarbon chains of surfactant molecules (Murudkar et al., 2015). This emission enhancement has been ascribed to the formation of ternary complex, ThT- $\gamma$-CD-hydrocarbon, which provides a rigid packing of the free space in the cyclodextrin cavity, leading to significant restriction to the torsional relaxation of ThT and thus yielding increase in emission intensity (Murudkar et al., 2015).

A similar host-guest complex of ThT with another supramolecular host, cucurbit[7]uril (CB7), has been employed as sensor for fluoride anion, when suitable amount of $\mathrm{Ca}^{2+}$ was added to the stoichiometrically mixed CB7 and ThT (CB7:ThT $=$ 2:1). This ternary supramolecular nanocapsule, $\mathrm{Ca}^{2+}$ CB7-ThT displayed excellent selectivity towards fluoride anions in complete aqueous solution. Importantly, the LOD of the fluoride ion, achieved using this system was 68 times lower than the maximum limit defined by Environmental Protection
Agency (EPA) which suggests the prospect of this fluoride sensor in real application (Zhu et al., 2014).

Recently, the interaction of ThT with another supramolecular host, sulphated $\beta-\mathrm{CD}$ (SCD), has been probed (Mudliar and Singh, 2016b). Interestingly, it was observed that this heavily negatively charged host molecule, SCD, induced the formation of emissive $\mathrm{H}$-aggregates of ThT instead of forming a conventional 1:1 inclusion complex with ThT. The formation of emissive $\mathrm{H}$ aggregates of ThT, upon interaction with SCD, was attributed to suppression of non-radiative torsional relaxation of ThT, which, in turn, activated the fluorescence channel, and hence ThT-SCD exhibited significant fluorescence enhancement as compared to ThT in bulk water. Most importantly, a distinctive enhanced red-shifted emission band at $545 \mathrm{~nm}$, in contrast to weakly emissive monomeric band at $490 \mathrm{~nm}$ band was observed. Appearance of this new emission band at $545 \mathrm{~nm}$, along with other changes in spectral properties of ThT, upon interaction with SCD, strengthened the hypothesis that SCD induced the formation of highly emissive ThT H-aggregates (Mudliar and Singh, 2016b).

In 2017, this ThT-SCD supramolecular dye aggregate template was explored to devise sensors for important biomolecules-arginine and lysine (Pettiwala and Singh, 2017). This sensor system was based on the principle that basic amino acids such as arginine and lysine would interact electrostatically with the anionic SCD which, may result in dissociation of ThT aggregates from SCD. This dissociation of ThTSCD assembly will, in turn, bring about modulations in monomer-aggregate equilibrium allowing ratiometric detection of arginine and lysine (Fig. 9). This proposition was well supported by absorbance, fluorescence CD spectroscopy and emission life time studies (Pettiwala and Singh, 2017).

In fluorescence measurements, it was evident that gradual addition of arginine to ThT-SCD complex led to a corresponding decrease in fluorescence intensity of the complex. This decrease in fluorescence intensity can be accounted to the disassembly of ThT H-aggregates from SCD surface as a result of electrostatic interaction and hydrogen bonding between arginine and SCD. Since the free ThT is weakly emissive in water, the dissociation of 


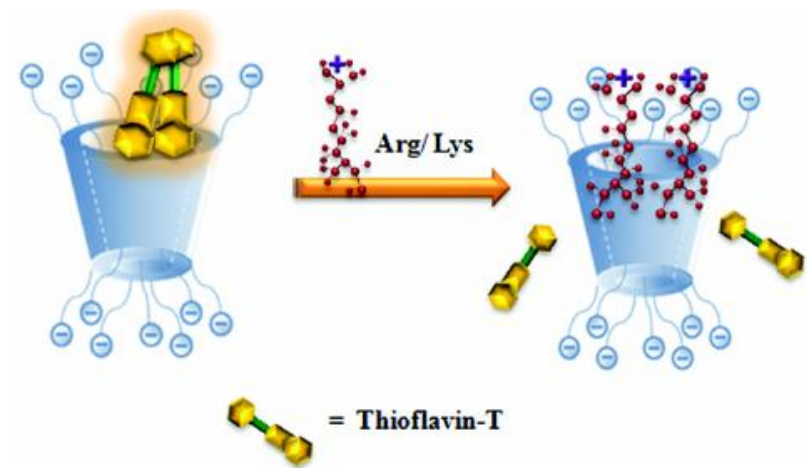

Fig. 9: Schematic illustration of arginine/lysine induced dissociation of ThT aggregates from SCD surface (Adapted from Pettiwala and Singh, 2017 with permission from American Chemical Society)

ThT H-aggregates from the surface of SCD towards monomeric form of ThT resulted in decrease of emission intensity. The shift in the population of ThTSCD aggregate form (highly emissive) to ThT monomer form (weakly emissive) on addition of arginine, allows ratiometric detection of arginine by monitoring the ratio of emission intensities at $545 \mathrm{~nm}$ (aggregate form) and $490 \mathrm{~nm}$ (monomeric form), thus yielding a quantitative estimation of arginine (Pettiwala and Singh, 2017).

In absorption studies, addition of arginine to ThTSCD complex resulted in a bathochromic shift of absorption spectra from $406 \mathrm{~nm}$ (ThT aggregate form) to $413 \mathrm{~nm}$ (ThT monomer). This red-shift in absorption spectra indicated the dissociation of ThT aggregates from SCD surface, and release of ThT monomer in bulk water. The excited-state lifetime results were in conjunction with fluorescence and absorbance studies wherein it was observed that addition of arginine in ThT-SCD results in faster excited-state lifetime, thus indicating the disruption of ThT-SCD complex and release of ThT monomer in solution (Pettiwala and Singh, 2017).

Similar changes in photophysical properties of ThT-SCD complex was observed on addition of lysine as both arginine and lysine contain positively charged side chains, hence their mode of interaction remains identical with ThT-SCD complex, based on electrostatic interactions and H-bonding. However, arginine shows marginally better response to supramolecular-dye aggregate platform than lysine. Moreover, it was demonstrated that arginine exhibits multi-wavelength distinct recognition pattern which differentiates it from lysine, using the current sensor system. More importantly, this sensor system based on ThT-SCD complex was able to detect arginine and lysine in biologically complex media like serum (Pettiwala and Singh, 2017).

Very recently, the ThT-SCD supramolecular dye aggregate assembly was further utilized to design a sensitive and discriminative platform for detection of two different classes of proteins (Pettiwala and Singh, 2018a). The extreme sensitivity of the photophysical properties of ThT towards its local microenvironment and previous reports of interaction between protein and cyclodextrins prompted us to investigate the ThTSCD supramolecular assembly as sensory system towards protein detection. The ThT-SCD system displayed a differential fluorescence response on interaction with proteins i.e., a turn-on signal towards non-metalloproteins and turn-off signal towards metalloproteins (Fig. 10). Crucially, the sensor system also enabled discrimination within same class of proteins using Principal component analysis (PCA) of the fluorescence patterns (Pettiwala and Singh, 2018a).

Thus, the precise and tuneable interactions of ThT with supramolecular host have facilitated researchers to design sensor platforms for important biomolecules, which in-near future can be used in practical applications.

\section{Thioflavin-T: Sensor for Heparin}

Heparin is the most widely used anticoagulant, administered to a patient undergoing surgical operations as well as for post-operational maintenance.

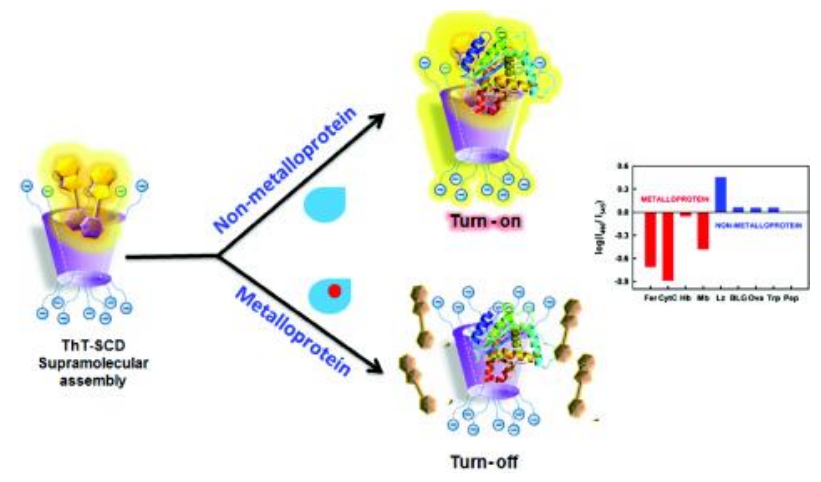

Fig. 10: Schematic representation of a supramolecular-ThT platform for detection and discrimination of 2 classes of proteins (Adapted from Pettiwala and Singh 2018a from Royal Society of Chemistry) 
However, an overdose of Heparin may lead to certain post-operative complications. This makes it inevitable to monitor the levels of Heparin in post-operative care. Recently, the spectroscopic investigation of the direct interaction between ThT and clinically important biomolecule, Heparin, has been reported which yielded unprecedented changes in the spectral features of ThT (Mudliar and Singh, 2016a). Accrediting to high negative charge density on the surface of Heparin, ThT molecules were made to form aggregate with a head-to-head arrangement i.e., H-aggregate. Generally, H-aggregates are poorly emissive in nature, however, ThT forms highly emissive $\mathrm{H}$-aggregates on Heparin, which enables a turn-on sensing mechanism for Heparin. In fluorescence titration of ThT with Heparin, a characteristic enhanced emission is noted at a large red shifted wavelength of $560 \mathrm{~nm}$ in contrast to a very weak emission band at $490 \mathrm{~nm}$. This largely shifted enhanced emission band centred at $560 \mathrm{~nm}$ is quite different compared to the usually observed enhanced emission band at $490 \mathrm{~nm}$ for ThT when in association with amyloid fibrils or Gquadruplex DNA. The $560 \mathrm{~nm}$ band is attributed to ThT aggregates formed on Heparin surface, and this proposition is further supported by evidence from absorption, excitation, CD spectroscopy and timeresolved fluorescence experiments. Both fluorescence and absorption measurement allowed detection of Heparin within range of $0-15 \mu \mathrm{M}$ with LOD value 18 $\mathrm{nM}$ and $26 \mathrm{nM}$ respectively enabling a dual mode of detection for Heparin. Furthermore, this sensor system demonstrated excellent selectivity towards Heparin compared to its structural analogues and contaminants namely Hyaluronic acid and Chondroitin sulphate. Also, this sensor system detected Heparin in biological complex media like fetal bovine serum and human serum-Heparin mixtures, thus exhibiting its practical utility. Further, ThT H-aggregates formed on the surface of Heparin were able to monitor the interaction of heparin with protamine which is its only clinically approved antidote. On addition of protamine to ThTHeparin solution a decrease in fluorescence at 560 nm was observed (Fig. 11). This decrease in fluorescence intensity was accounted for disassembly of ThT aggregates from Heparin surface, as a result of electrostatic interaction between positively charged protamine and negatively charged Heparin. Thus, ThT, by the virtue of changes in its photophysical properties, was able to sense Heparin and the same platform was used to probe interaction of protamine with Heparin (Mudliar and Singh, 2016a).

Based on similar concept of dissociation of ThT $\mathrm{H}$ aggregates from Heparin surface upon interaction with basic amino acids, a sensor for arginine and lysine was devised (Pettiwala and Singh, 2018b). The strong electrostatic interactions and hydrogen bonding between Heparin and basic amino acids, lead to changes in photophysical properties of ThT, which enabled ratiometeric detection of arginine and lysine (Fig. 12). Moreover, this sensory ensemble allowed dual mode of detection for basic amino acids that is both fluorimetry and colorimetry, with LOD values as low as $1 \mu \mathrm{M}$. Thus, this sensing scheme based on ThT provides a facile and selective platform for detection of basic amino acids (Pettiwala and Singh, 2018b).
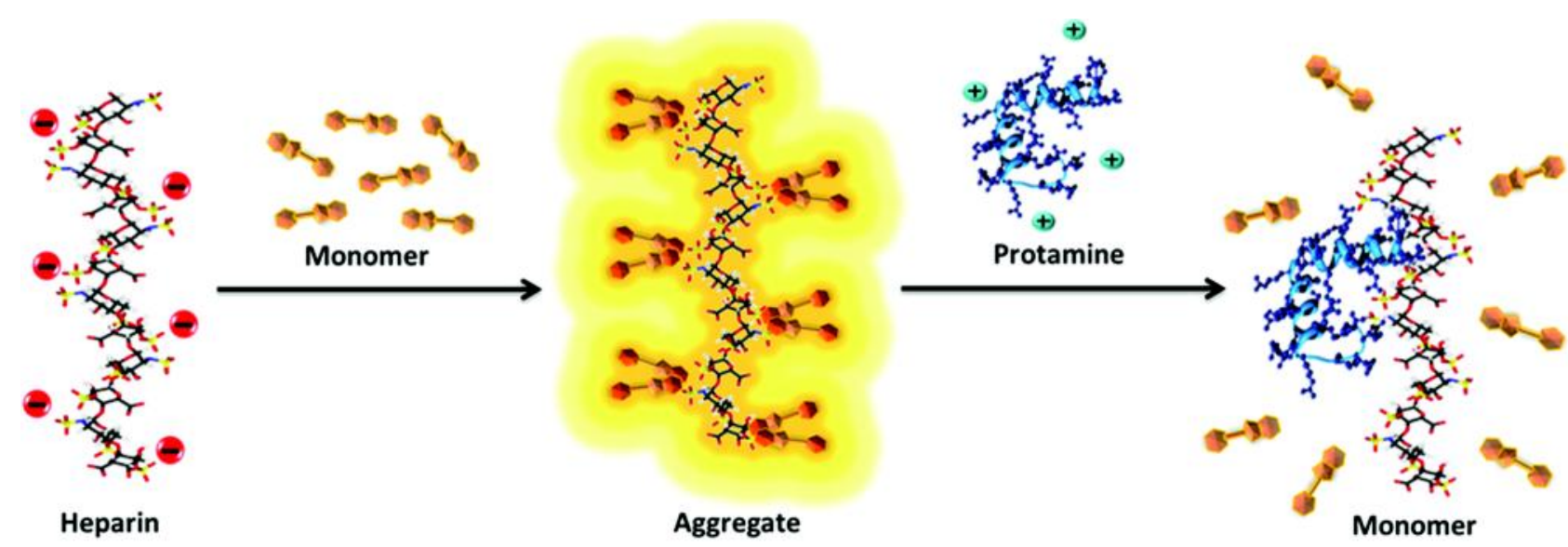

Fig. 11: Schematic representation of Heparin Induced ThT aggregates and its Dissociation upon heparin"Protamine Interaction (Adapted from Mudliar and Singh 2016a with permission from American Chemical Society) 


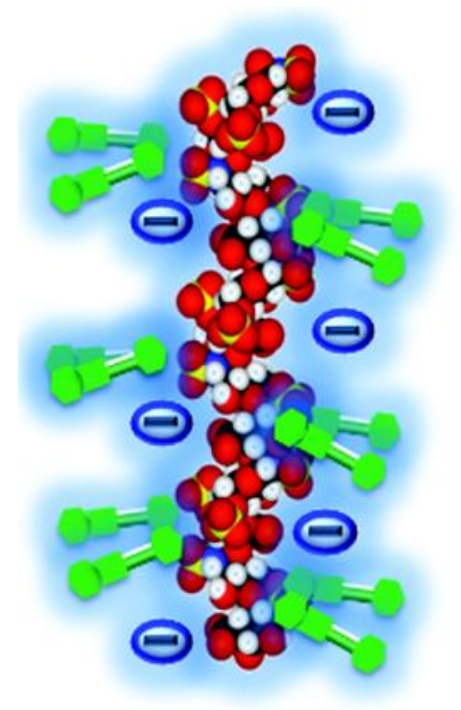

\section{ThT-Heparin complex}

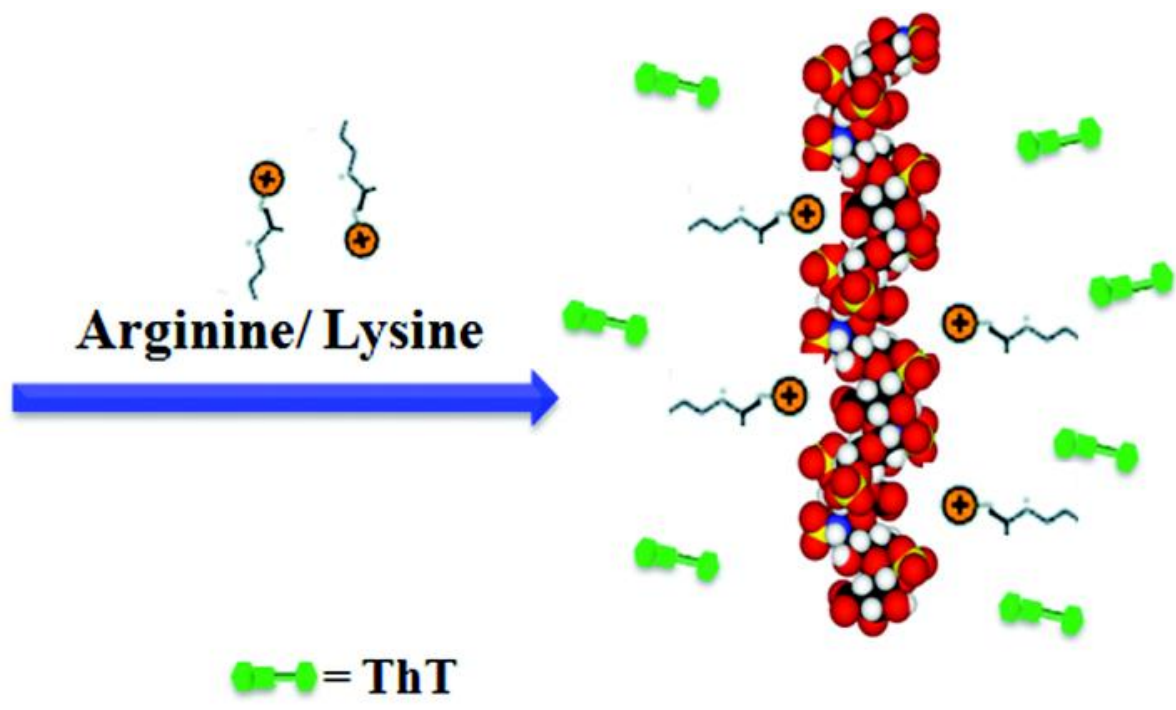

ThT Monomer

Fig. 12: Schematic representation of arginine/lysine induced dissociation of ThT aggregates from Heparin surface (Adapted from Pettiwala and Singh 2018b with permission from Elsevier)

ThT has also shown to act as sensor for few enzymes (Ma et al., 2016), microRNA (Fan et al., 2017) and aptamers (Wang et al., 2016) based on the common mechanism of modulation of its fluorescence property on interacting with different target analytes. Although these sensors have certain limitations, however they might act as potential sensory systems for real-time applications in biomedical and clinical areas.

At the end, we would like to add some general remarks on the usage of this versatile probe molecule with respect to the practical aspects in sensing applications. Since ThT belongs to the class of molecular rotors, whose photophysical properties are quite sensitive to the temperature, thus, the performance of ThT based sensors are prone to be affected by the increase in temperature of the environment. However, the photostability of ThT is quite good, and moreover, ThT is also stable in a wide $\mathrm{pH}$ range of 2 to 9 at room temperature, which assures the reproducibility of ThT based sensors. Additionally, ThT is available commercially at a very economical price which avoids the time-consuming and tedious synthetic efforts, generally required for the synthesis of a sensor probe, and thus this can largely impact the usage of this probe in real-life applications. These useful attributes of ThT makes it a very popular probe for sensing applications which is evidenced by a large number of literature reports on its sensing applications in recent past.

\section{Conclusion}

In this review article, we attempted to cover all the recent works which outline the application of Thioflavin-T as fluorescent sensor for detection of various target analytes. All of the aforementioned chemo and biosensing application of ThT are based on either turn-on or turn-off fluorescent mechanism of dye, which arises because of alterations in its photophysical properties in response to the changes in the micro-environment of the dye. The initial section of the review article is dedicated to the photophysics of ThT followed by the common sensory application of ThT to detect amyloid fibrils. Next, we have discussed the detailed interactions of ThT with various forms of DNA including G-quadruplex DNA and supramolecular hosts which act as sensor platforms for metal-ions, amino acids, biothiols, Thrombin and so on. Finally, we have outlined the interaction of Thioflavin-T with Heparin, where ThT aggregates, instead of ThT monomers works as sensing ensemble. These sensor platforms based on Thioflavin-T are highly sensitive, simple and cost-effective compared to other conventional methods employed for detection 
of clinically relevant molecules. Through this short review of Thioflavin-T sensory applications, we hope to stimulate new ideas and constructs for detection of other biologically important molecules.

\section{References}

Amdursky N, Erez Y and Huppert D (2012) Molecular Rotors: What Lies Behind the High Sensitivity of the ThioflavinT Fluorescent Marker Acc Chem Res 45 1548-1557

Amdursky N, Gepshtein R, Erez Y and Huppert D (2011a) Temperature Dependence of the Fluorescence Properties of Thioflavin-T in Propanol, a Glass-Forming Liquid $J$ Phys Chem A 115 2540-2548

Amdursky N, Gepshtein R, Erez Y, Koifman N, Huppert D (2011b) Pressure Effect on the Nonradiative Process of Thioflavin-T J Phys Chem A 115 6481-6487

Bai D, Ji D, Shang J, Hu Y, Gao J, et al. (2017) A Rapid Biosensor for Highly Sensitive Protein Detection based on Gquadruplex-Thioflavin T Complex and Terminal Protection of Small Molecule-linked DNA Sensors and Actuators B: Chemical 252 1146-1152

Biancalana M and Koide S (2010) Molecular Mechanism of Thioflavin-T Binding to Amyloid Fibrils Biochim Biophys Acta 1804 1405-1412

Biancardi A, Biver T, Burgalassi A, Mattonai M, Secco F, et al. (2014) Mechanistic Aspects of Thioflavin-T Selfaggregation and DNA Binding: Evidence for Dimer Attack on DNA Grooves Phys Chem Chem Phys 16 20061-20072

Biancardi A, Biver T and Mennucci B(2017) Fluorescent Dyes in the Context of DNA-binding: The Case of Thioflavin T Int J Quantum Chem 118 1-6

Biju V (2014) Chemical modifications and Bioconjugate Reactions of Nanomaterials for Sensing, Imaging, Drug Delivery and Therapy Chem Soc Rev $\mathbf{4 3}$ 744-764

Chiti F and Dobson C M (2006) Protein Misfolding, Functional Amyloid, and Human Disease Annu Rev Biochem 75 333366

Chu T, Li Z, Liu X and Wang X (2007) In Vivo Evaluation of the Nitroimidazole-Based Thioflavin-T Derivatives as Cerebral Ischemia Markers International Journal of Biomedical Imaging 2007 49791-49796

Cosnier S and Mailley P (2008) Recent Advances in DNA Sensors

\section{Acknowledgement}

We sincerely acknowledge all the collaborators and co-authors of our published articles which are cited in this review article. We also sincerely thank Dr. H. Pal and Dr. P. D. Naik for their constant encouragement and support throughout. AMP and NHM are thankful to Dr. A. Khanna, NMIMS, Mumbai for her encouragement.

\section{Analyst 133 984-991}

Fan T, Mao Y, Liu F, Zhang W, Yin J, et al. (2017) Dual Signal Amplification Strategy for Specific Detection of Circulating microRNAs Based on Thioflavin T Sens Actuators B 249 $1-7$

Faverie A R, Gue A, Bedrat A, Yatsuny L A and Mergny J-L (2014) Thioflavin T as a Fluorescence Light-up Probe for G4 Formation Nucleic Acids Res 42 1-8

G Ventimiglia and S Petralia (2013) Recent Advances in DNA Microarray Technology : An Overview on Production Strategies and Detection Methods Bio Nano Sci 3 428-450

Gabelica V, Maeda R, Fujimoto T, Yaku H, Murashima T, et al. (2013) Multiple and Cooperative Binding of Fluorescence Light-up Probe Thioflavin T with Human Telomere DNA G-Quadruplex Biochemistry 52 5620-5628

Ge J, Li X, Jiang J and Yu R (2014) A Highly Sensitive Label-free Sensor for Mercury Ion $(\mathrm{Hg} 2+)$ By Inhibiting Thioflavin $\mathrm{T}$ as DNA G-quadruplexes Fluorescent Inducer Talanta $12285-90$

Gellert M, Lipsett M N and Davies D R (1962) Helix Formation by Guanylic Acid Proc Natl Acad Sci 48 2013-2018

Gestwicki J E, Crabtree G R and Graef I A (2004) Harnessing Chaperones to Generate Small-molecule Inhibitors of Amyloid b Aggregation Science 306 865-869

Glenn G and Andreou L-V (2013) Analysis of DNA by Southern Blotting Meth Enzymol 529 47-63

Groenning M, Norrman M, Flink J M, Weert M v d, Bukrinsky J T, et al. (2007a) Binding Mode of Thioflavin T in Insulin Amyloid Fibrils J Struct Biol 159 483-497

Groenning M, Olsen L, Weert M v d, Flink J M, Frokjaer S, et al. (2007b) Study on the binding of Thioflavin T to betasheet-rich and non-beta-sheet cavities J Struct Biol 158 358-369

Guo J J, Wang J C, Zhao J Q, Guo Z L and Zhang Y Z (2016) Ultrasesitive Multiplexed Immunoassay for Tumor Biomarkers based on DNA Hybridisation Chain Reaction ACS Appl Mater Interfaces 8 6898-6904 
Guo K, Pourpak A, Beetz-Rogers K, Gokhale V, Sun D, et al. (2007) Formation of Pseudosymmetrical G-Quadruplex and $\mathrm{i}$-Motif Structures in the Proximal Promoter Region of the RET Oncogene J Am Chem Soc 129 10220-10228

Haidekker M A, Brady T P, Lichlyter D and Theodorakis E A (2005) Effects of Solvent Polarity and Solvent Viscosity on the Fluorescent Properties of Molecular Rotors and Related Probes. Bioorg Chem 33 415-425

Haidekker M A, Nipper M, Mustafic A, Lichlyter D, Dakanali M, et al. (2010) Dyes with Segmental Mobility: Molecular Rotors In: Advanced Fluorescence Reporters in Chemistry and Biology I. Fundamentals and Molecular Design (Demchenko AP Ed) pp 267-308 Springer-Verlag

Harel M, Sonoda L K, Silman I, Sussman J L and Rosenberry T L (2008) Crystal Structure of Thioflavin T Bound to the Peripheral Site of Torpedo californica Acetylcholinesterase Reveals How Thioflavin T Acts as a Sensitive Fluorescent Reporter of Ligand Binding to the Acylation Site $J$ Am Chem Soc 130 7856-7861

Huang V, Huang W-H and Sun F-Z (2010) Modified Agarose Gel for High Performance Southern Blotting of Short DNA Fragments BioTech 49 580-581

Ilanchelian M and Ramaraj R (2004) Emission of Thioflavin T and its Control in the Presence of DNA Journal of Photochemistry and Photobiology A: Chemistry 162 129137

Ivanova M I, Sievers S A, Sawaya M R, Wall J S and Eisenberg D (2009) Molecular Basis for Insulin Fibril Assembly Proc Natl Acad Sci USA 106 18990-18995

Ji H X, Yan F, Lei J P and Ju H X (2012) Ultrasensitive Electrochemical Detection of Nucleic Acids by Template Enhanced Hybridization Followed with Rolling Circle Amplification Anal Chem 84 7166-7171

Khurana R, Coleman C, Ionescu-Zanetti C, Carter S A, Krishna $\mathrm{V}$, et al. (2005) Mechanism of thioflavin T binding to amyloid fibrils J Struct Biol 151 229-238

Kitts C C and Bout D A V (2009) Near-Field Scanning Optical Microscopy Measurements of Fluorescent Molecular Probes Binding to Insulin Amyloid Fibrils J Phys Chem B 113 12090-12095

Klunk W E, Wang Y, Huang G F, Debnath M L, Holt D P, et al. (2001) Uncharged thioflavin-T derivatives bind to amyloid-beta protein with high affinity and readily enter the brain Life Sci 69 1471-1484

Krebs M R H, Bromley E H C and Donald A M (2005) The binding of thioflavin-T to amyloid fibrils: localisation and implications J Struct Biol 149 30-37
Kumar S, Singh A K, Krishnamoorthy G and Swaminathan R (2008) Thioflavin-T displays enhanced fluorescence selectively inside anionic micelles and mammalian cells $J$ Fluoresc 18 1199-1205

Lee J, Patil S P, Fhayli K S A and Khashab N M (2015) Probing Structural Changes of Self Assembled i-motif DNA Chem Commun 51 3747-3749

LeVine H, III (1993) Thioflavine-T Interaction with Synthetic Alzheimer's Disease b-amyloid Peptides: Detection of Amyloid Aggregation in Solution Protein Sci 2 404-410

Li X, Gan L, Ou Q, Zhang X, Cai X, et al. (2015) Enzyme-free and Label-free Fluorescence Sensor for the Detection of Liver Cancer Related Short Gene Biosensors and Bioelectronics 66 399-404

Liu L, Shao Y, Peng J, Huang C, Liu H, et al. (2014) Molecular Rotor-Based Fluorescent Probe for Selective Recognition of Hybrid G-quadruplex and as a K+ Sensor Anal Chem 86 $1622-1631$

Liu L, Shao Y, Peng J, Liu H and Zhang L (2013) Selective Recognition of ds-DNA Cavities by a Molecular Rotor: Switched Fluorescence of Thioflavin T Mol Bio Syst 9 2512-2519

Liu X, Hua X, Fan Q, Chao J, Su S, et al. (2015) Thioflavin T as an Efficient G-quadruplex Inducer for Highly Sensitive Detection of Thrombin Using a new FRET System ACS Appl Mater Interfaces 30 16458-16465

Ma C, Liu H, Li W, Chen H, Jin S, et al. (2016) Label-free Monitoring of DNA Methyltransferase Activity based on Terminal Deoxynucleotidyl Transferase using a Thioflavin T Probe Mol and Cell Probes 2 118-121

Maezawa I, Hong H-S, Liu R, Wu C-Y, Cheng R-H, et al. (2008) Congo red and Thioflavin-T Analogs Detect Ab Oligomers J Neurochemistry 104 457-468

Maskevich AA, Stsiapura V I, Kuzmitsky V A, Kuznetsova I M, Povarova O I, et al.(2007) Spectral Properties of Thioflavin-T in Solvents with Different Dielectric Properties and in a Fibril-incorporated Form J Proteome Res 6 1392-1401

Mergny J L and Lacroix L (2009) UV Melting of G-Quadruplexes Curr Protoc Nucleic Acid Chem 37

Mergny J L, Li J, Lacroix L, Amrane S, Chaires J B (2005) Thermal Difference Spectra: A Specific Signature for Nucleic Acid Structures Nucleic Acids Res 33 138-144

Mohanty J, Barooah N, Dhamodharan V, Harikrishna S, Pradeepkumar P I, et al. (2013) Thioflavin T as an Efficient Inducer and Selective Fluorescent Sensor for the Human Telomeric G-Quadruplex DNA J Am Chem Soc 135367 - 
376

Mora A K, Murudkar S, Alamelu A, Singh P K, Chattopadhyay S, et al.(2016) Benzothiazole-Based Neutral Ratiometric Fluorescence Sensor for Amyloid Fibrils Chem Eur $\mathbf{2 2}$ 16505-16512

Mudliar N H, Sadhu B, Pettiwala A M and Singh P K (2016) Evaluation of An Ultrafast Molecular Rotor, Auramine O, as Fluorescent Amyloid Marker J Phys Chem B 120 1049610507

Mudliar N H, Singh P K (2016a) Emissive H-Aggregates of an Ultrafast Molecular Rotor: A Promising Platform for Sensing Heparin Appl Mater Interfaces 8 31505-31509

Mudliar N H and Singh P K (2016b) Fluorescent H-aggregates Hosted by a Charged Cyclodextrin Cavity Chem Euro J 22 7394-7398

Murudkar S, Mora A K, Jakka S, Singh P K and Nath S (2014) Ultrafast molecular rotor based DNA sensor: An insight into the mode of interaction J Photochem Photobio A 295 $17-25$

Murudkar S, Mora A K, Singh P K, Bandyopadhyay T and Nath S (2015) An ultrafast molecular rotor based ternary complex in a nanocavity: a potential "turn on" fluorescence sensor for the hydrocarbon chain Phys Chem Chem Phys 1756915703

Murudkar S, Mora A K, Singh P K and Nath S (2012) Ultrafast Molecular Rotor: An Efficient Sensor for Premelting of Natural DNA Chem Commun 48 5301-5303

Naik L R, Naik A B and Pal H (2009) Steady-State and TimeResolved Emission Studies of Thioflavin-T J Photochem Photobiol A 204 161-167

Nelson R and Eisenberg D (2006) Recent atomic models of amyloid fibril structure Curr Opin Struct Biol 16 260-265

Noel S, Cadet S, Gras E and Hureau C(2013) The benzazole scaffold: A SWAT to combat Alzheimer's disease Chem Soc Rev 42 7747-7762

Ono A, Torigoe H, Tanaka Y and Okamoto I (2011) Binding of Metal Ions by Pyrimidine Base Pairs in DNA Duplexes Chem Soc Rev 40 5855-5866

Pettiwala A M and Singh P (2018a) A Supramolecular Assembly Enables Discrimination Between Metalloproteins and Nonmetalloproteins Chem Commun 54 4537-4540

Pettiwala A M and Singh P K (2017) Supramolecular Dye Aggregate Assembly Enables Ratiometric Detection and Discrimination of Lysine and Arginine in Aqueous Solution ACS Omega 2 8779-8787

Pettiwala A M and Singh P K (2018b) A Molecular Rotor based Ratiometric Sensor for Basic Amino Acids Spectrochim
Acta A 188 120-126

Phan A T, Kuryavyi V, Luu K N and Patel D J (2007) Structure of Two Intramolecular G-quadruplexes Formed by Natural Human Telomere Sequences in $\mathrm{K}+$ Solution Nucleic Acids Res 35 6517-6525

Raj C R and Ramaraj R (2001) Emission of Thioflavin T and its Off-On Control in Polymer Membranes Photochem Photobio $74752-759$

Rettig W, Lapouyade R and Lakowicz J R (1994) Topics in Fluorescence Spectroscopy, Probe Design and Chemical Sensing 4 109-149

Rodrýiguez-Rodrýiguez C, Groot N S d, Rimola A, AlvarezLarena A, Lloveras V, et al. (2009) Design, Selection, and Characterization of Thioflavin-Based Intercalation Compounds with Metal Chelating Properties for Application in Alzheimer's Disease J Am Chem Soc 131 1436-1451

Sabate R, Lascu I and Saupe S J (2008) On the binding of Thioflavin-T to HET-s amyloid fibrils assembled at $\mathrm{pH} 2$ J Struct Biol 162 387-396

Sabate R and Saupe S J (2007) Thioflavin T fluorescence anisotropy: An alternative technique for the study of amyloid aggregation Biochem Biophys Res Comm 360135 138

Sassolas A, Leca-Bouvier B D and Blum L J (2008) DNA biosensors and microarrays Chem Rev 108 109-139

Schaerli Y, Wootton R C, Robinson T, Stein V, Dunsby C, et al. (2009) Continuous-flow Polymerase Chain Reaction of Single-copy DNA in Microfluidic Microdroplets Anal Chem 81 302-306

Schirra R Dye aggregation in freezing aqueous solutions (1985) Chem Phys Letts 119 463-466

Sen P, Fathima S, Ahmad B, Khan R H Interactions of ThioflavinT with Serum Albumins: Spectroscpoc Analyses Spectrochim Acta A 200974 94-99

Singh P K, Jakka S, Mora A K and Nath S (2012) Probing the DNA-Ionic Liquid Interaction Using an Ultrafast Molecular Rotor J Photochem Photobiol A 246 16-22

Singh P K, Kumbhakar M, Pal H and Nath S (2009) Ultrafast Torsional Dynamics of Protein Binding Dye Thioflavin-T in Nanoconfined Water Pool J Phys Chem B 113 85328538

Singh P K, Kumbhakar M, Pal H and Nath S (2010a) Ultrafast Bond Twisting Dynamics in Amyloid Fibril Sensor J Phys Chem B 114 2541-2546

Singh P K, Kumbhakar M, Pal H and Nath S (2010b) Viscosity Effect on the Ultrafast Bond Twisting Dynamics in an 
Amyloid Fibril Sensor: Thioflavin-T J Phys Chem B 114 5920-5927

Singh P K, Kumbhakar M, Pal H and Nath S (2011a) Confined Ultrafast Torsional Dynamics of Thioflavin-T in a Nanocavity Phys Chem Chem Phys 13 8008-8014

Singh P K, Kumbhakar M, Pal H and Nath S (2011b) A Nanoconfined Charged Layer Defies the Principle of Electrostatic Interaction Chem Commun 47 6912-6914

Singh P K, Mora A K and Nath S (2015a) Ultrafast Fluorescence Spectroscopy Reveals a Dominant Weakly-emissive Population of Fibril Bound Thioflavin-T Chem Commun 51 14042-14045

Singh P K, Mora A K and Nath S (2015b) Ultrafast Torsional Relaxation of Thioflavin-T in Tris (pentafluoroethyl) trifluorophosphate (FAP) Anion-Based Ionic Liquids $J$ Phys Chem B 119 14252-14260

Singh P K, Mora A K and Nath S(2016) Free volume dependence of an ionic molecular rotorin Fluoroalkylphosphate (FAP) based ionic liquids Chem Phys Lett 644 296-301

Singh P K, Murudkar S, Mora A K and Nath S (2015c) Ultrafast Torsional Dynamics of Thioflavin-T in an Anionic Cyclodextrin Cavity J Photochem Photobio, A 298 40-48

Singh P K and Nath S (2012) Ultrafast Torsional Dynamics in Nanoconfined Water Pool: Comparison between Neutral and Charged Reverse Micelles J Photochem Photobiol, A 248 42-49

Singh P K and Nath S (2013) Molecular Recognition Controlled Delivery of a Small Molecule from a Nanocarrier to Natural DNA J Phys Chem B 117 10370-10375

Srivastava A, Singh P K, Kumbhakar M, Mukherjee T, Chattopadyay S, et al. (2010) Identifying the bond responsible for the fluorescence modulation in amyloid fibril sensor Chem Eu J 169257

Stsiapura V I, Maskevich A A, Kuzmitsky V A, Turoverov K K and Kuznetsova I M (2007) Computational Study of Thioflavin T Torsional Relaxation in the Excited State $J$ Phys Chem A 111 4829-4835

Stsiapura V I, Maskevich A A, Kuzmitsky V A, Uversky V N, Kuznetsova I M, et al. (2008) Thioflavin T As a Molecular Rotor: Fluorescent Properties of Thioflavin T in Solvents with Different Viscosity J Phys Chem B 112 15893-15902

Stsiapura V I, Maskevich AA, Tikhomirov S A and Buganov O V (2010) Charge Transfer Process Determines Ultrafast Excited State Deactivation of Thioflavin $\mathrm{T}$ in LowViscosity Solvents J Phys Chem A 114 8345-8350

Sulatskaya A I, Kuznetsova I M and Turoverov K K (2012) Interaction of Thioflavin $\mathrm{T}$ with Amyloid Fibrils:
Fluorescence Quantum Yield of Bound Dye J Phys Chem B 116 2538-2544

Sulatskaya A I, Maskevich A A, Kuznetsova I M, Uversky V N and Turoverov K K (2010) Fluorescence Quantum Yield of Thioflavin T in Rigid Isotropic Solution and Incorporated into the Amyloid Fibrils PLoS One 5 1-7

Tassone F, Pan R, Amiri K, Taylor A K and Hagerman P J A (2008) Rapid Polymerase Chain Reaction-based Screening Method for Identification of all Expanded Alleles of the Fragile X (FMR1) Gene in Newborn and High-risk Populations J Mol Diagnos 10 43-49

Tong L-1, Li L, Chen Z, Wang Q and Tang B (2013) Stable labelfree Fluorescent Sensing of Biothiols Based on ThT Direct Inducing Conformation-specific G-quadruplex Biosens Bioelectron 49 420-425

Vetri V, Canale C, Relini A, Librizzi F, Militello V, et al.(2007) Amyloid fibrils formation and amorphous aggregation in concanavalin A Biophys Chem 125 184-190

Voropai E S, Samtsov M P, Kaplevskii K N, Maskevich A A, Stepuro V I, et al. (2003) Spectral Properties of Thioflavin $\mathrm{T}$ and its Complexes with Amyloid Fibrils J Appl Spec 70 868-874

Wang H, Peng P, Liu S and Li T (2016) Thioflavin T Behaves as an Efficient Fluorescent Ligand for Label-free ATP Aptasensor Anal Bioanal Chem 28 7927-7934

Wang Y, Geng F, Cheng Q, Xu H and Xu M (2011) Oligonucleotide-based Label-free $\mathrm{Hg} 2+$ Assay with a monomer-excimer Fluorescence Switch Analyst 1364284 4288

Wu Y and Guo L (2014) Photoelectrochemical Sensors for the Detection of DNA Damage Prog Chem 26 1-9

Xu H, Geng F, Jiangd X, Shao Y, Wang Y, et al. (2018a) Design of Metal-ion-triggered Assembly of Label-free Split Gquadruplex/duplex DNA for Turn-on Detection of $\mathrm{Hg} 2+$ in Fetal Calf Serum Sensors and Actuators B: Chemical 255 1024-1030

Xu S, Li Q, Xiang J, Yang Q, Sun H, et al. (2018b) Thioflavin T as an Efficient Fluorescence Sensor for Selective Recognition of RNA G-quadruplexes Sci Rep 6 1-9

$\mathrm{Xu}$ Y and Sugiyama H (2006) Formation of the G-quadruplex and i-motif Structures in Retinoblastoma Susceptibility Genes (Rb) Nucleic Acids Res 34 949-954

Yang X, Wei W, Jiang J, Shen G and Yu R (2016) Conformational switching of G-quadruplexes as a label-free platform for the fluorescence detection of $\mathrm{Ag}+$ and biothiols Anal Methods 8311-315

Yao J, Yang M and Duan Y (2014) Chemistry, Biology, and 
Medicine of Fluorescent Nanomaterials and Related Systems: New Insights into Biosensing, Bioimaging, Genomics, Diagnostics, and Therapy Chem Rev 114 61306178

Zhou J, Wei C, Jia G, Wang X, Feng Z, et al. (2010) Formation of i-motif structures at Neutral and Slightly Alkaline $\mathrm{pH} \mathrm{Mol}$ Bio Syst 6 580-586
Zhu J, Li C, Liu S, Liu Z, Li Y, et al. (2014) Calcium-stimulusresponsive cucurbit[7] uril-thioflavin $\mathrm{T}$ supramolecular nanocapsule for fluoride sensing and logic operation Sens Actuators, B 198 255-259. 